Pacific Journal of Mathematics

EXPANDED RADICAL IDEALS AND SEMIREGULAR IDEALS 


\section{EXPANDED RADICAL IDEALS AND SEMIREGULAR IDEALS}

\section{HOCHSTER}

Let $R=K\left[x_{1}, \cdots, x_{n}\right]$ be a polynomial ring, where $K$ is a field of characteristic zero, and embed $R$ in the polynomial ring $S=K\left[y_{i j}: 1 \leqq i \leqq n-1,1 \leqq j \leqq n\right]$ by mapping $x_{j}$ to the minor of the matrix $\left[y_{i j}\right]$ obtained by deleting the $j^{\text {th }}$ column. Let $I$ be a homogeneous radical ideal of $R$. It will be shown that if $I S$ is radical, then $I$ is semiregular, that is, $R / I$ is Cohen-Macaulay. Several other related results will be established, in which the fact that certain expanded radical ideals remain radical either implies, or is implied by, the fact that certain other ideals are semiregular. Each one of these results has some connection with invariant theory.

We shall now give a more detailed summary of the main results of the paper. If $R$ is a finitely generated $K$-algebra, where $K$ is a field, we shall say that an ideal $I$ of $R$ is absolutely radical (over $K$ ) if for each extension field $L$ of $K, L \bigotimes_{K} I$ is radical in $L \bigotimes_{K} R$. Of course, it suffices that $L \otimes_{K} I$ be radical when $L$ is a maximal purely inseparable extension of $K$, or any larger field, e.g. an algebraic closure of $K$.

THEOREM 1. Let $K$ be a field, and let $S$ be a graded Noetherian Cohen-Macaulay K-algebra which is flat over the $K$-subalgebra $R$ generated by the forms $f_{1}, \cdots, f_{m}$, where $f_{1}, \cdots, f_{m}$ have positive degree. (We do not require that $S$ be generated by its 1-forms.) Suppose that that $\left(f_{1}, \cdots, f_{m}\right)$ is an absolutely radical ideal of $S$. Then for every absolutely radical ideal $I$ of $R$, the expanded ideal IS is absolutely radical in $S$.

In particular, if $S$ is a polynomial ring over $K$ (the indeterminates need not be assigned degree 1 ), and $f_{1}, \cdots, f_{m}$ is an $R$-sequence of forms in $S$ which generates an absolutely radical ideal of $S$, then $S$ is flat over $K\left[f_{1}, \cdots, f_{m}\right]$ and the result holds.

We note that this theorem generalizes, in a certain direction, an invariant-theoretic result of Kostant, [6] Theorem 14, p. 388.

We point out that the hypothesis implies that $\left(f_{1}, \cdots, f_{m}\right)$ is a semiregular ideal of $S$, which plays an important role in the proof.

It is also worth noting that from the point of view of testing an ideal for being absolutely radical, an $R$-sequence of polynomial forms which generates an absolutely radical ideal is as good as a sequence of indeterminates, in the sense of the following: 
CoROLlary. Let $f_{1}, \cdots, f_{m}$ be an $R$-sequence of forms in the polynomial ring $K\left[x_{1}, \cdots, x_{n}\right]$ which generates an absolutely radical ideal. Let $g_{1}\left(y_{1}, \cdots, y_{m}, z_{1}, \cdots, z_{p}\right), \cdots, g_{t}\left(y_{1}, \cdots, y_{m}, z_{1}, \cdots, z_{p}\right)$ be polynomials in $K\left[y_{1}, \cdots, y_{m}, z_{1}, \cdots, z_{p}\right]$ which generate an absolutely radical ideal. Then $g_{1}\left(f_{1}, \cdots, f_{m}, z_{1}, \cdots, z_{p}\right), \cdots, g_{t}\left(f_{1}, \cdots, f_{m}, z_{1}, \cdots, z_{p}\right)$ generate an absolutely radical ideal of $K\left[x_{1}, \cdots, x_{n}, z_{1}, \cdots, z_{p}\right]$.

The corollary follows from the last statement in Theorem 1 (applied to the $R$-sequence $f_{1}, \cdots, f_{m}, z_{1}, \cdots, z_{p}$ of $\left.K\left[x_{1}, \cdots, x_{m}, z_{1}, \cdots, z_{p}\right]\right)$.

THEOREM 2. Let $K$ be a field of characteristic zero, and let $I$ be a homogeneous radical ideal of the polynomial ring $K\left[x_{1}, \cdots, x_{n}\right]$. Suppose $I=\left(f_{1}, \cdots, f_{m}\right)$. Let $\left[y_{i j}\right]$ be an $n-1$ by $n$ matrix of new indeterminates, and let $\Delta_{i}$ be the $n-1$ by $n-1$ minor of $\left[y_{i j}\right]$ which results from deleting the $i^{\text {th }}$ column, $1 \leqq i \leqq n$. Then a sufficient condition for $I$ to be semiregular (三 perfect in this case) is that $f_{1}\left(\Delta_{1}, \cdots, \Delta_{n}\right), \cdots, f_{m}\left(\Delta_{1}, \cdots, \Delta_{n}\right)$ generate a radical ideal in $K\left[y_{i j}: i, j\right]$.

In other words, it is sufficient that $I$ expand to a radical ideal under the injective $K$-homomorphism $K\left[x_{1}, \cdots, x_{n}\right] \rightarrow K\left[y_{i j}: i, j\right]$ which takes $x_{i}$ to $\Delta_{i}, 1 \leqq i \leqq n$.

The proof of Theorem 2 depends on some invariant-theoretic results and on the following:

Lemma. Let $K$ be a field, and let $I$ be a homogeneous radical ideal in a reduced graded Noetherian Cohen-Macaulay K-algebra $R$. Let $x_{1}, \cdots, x_{n}$ be any elements of $R$ such that the radical of $\left(x_{1}, \cdots, x_{n}\right)$ is the irrelevant maximal ideal $M$ of $R$. Let $d=\operatorname{dim} R / I$, and let $t_{i j}$ be $n(d-1)$ new indeterminates over $R$. Then $I$ is semiregular if and only if for each $i, 1 \leqq i \leqq d-1$, the ideal

$$
I+\left(t_{11} x_{1}+\cdots+t_{1 n} x_{n}, \cdots, t_{i 1} x_{1}+\cdots+t_{i n} x_{n}\right)
$$

is radical in $R\left[t_{i j}: i, j\right]$.

The condition given in Theorem 2 is not necessary. However, one might hope that some special classes of perfect radical homogeneous ideals in $K\left[x_{1}, \cdots, x_{n}\right]$ would expand to radical ideals in $K\left[y_{i j}\right]$. This is true at least for the ideals in $K\left[x_{1}, \cdots, x_{n}\right]$ which are generated by linear combinations of $x_{1}, \cdots, x_{n}$.

Let us say that a $K$-vector subspace $V$ of a $K$-algebra $R$ is linearly radical (over $K$ ) if for any field $L \supset K$ and for each finite set of elements $f_{1}, \cdots, f_{m} \in L \bigotimes_{K} V,\left(f_{1}, \cdots, f_{m}\right)$ is radical ideal in $L \bigotimes_{K} R$. A trivial example is obtained by letting $V$ be the space of 1 -forms in $K\left[x_{1}, \cdots, x_{n}\right]$. Less trivially, we have: 
THEOREM 3. (a) Let $\left[y_{i j}\right]$ be an $r$ by $s$ matrix of indeterminates over the field $K$, where $r \leqq s$, and let $V$ be the subspace of $K\left[y_{i j}: i, j\right]$ spanned by the $r$ by $r$ minors of $\left[y_{i j}\right]$. If $s=r+1$ then $V$ is linearly radical. If $r=2$, it is at least true that any subject of the 2 by 2 minors generates a radical ideal.

(b) Let $\Lambda$ be a partially ordered set and suppose that for each $\lambda \in \Lambda$ the set $\{u \in \Lambda: \mu \leqq \lambda\}$ is finite and totally ordered. Let $\left\{U_{\lambda}: \lambda \in \Lambda\right\}$ be a family of matrices of indeterminates, all entries distinct, indexed by $\Lambda$, and suppose that (1) if $\lambda$ is minimal in $\Lambda$ then $U_{\lambda}$ is a one-rowed matrix, and (2) for each $\lambda \in \Lambda$, if $\lambda_{1}<\lambda_{2}<\cdots<\lambda_{n}=\lambda$ are the elements of $\{\mu \in \Lambda: \mu \leqq \lambda\}$, then the product matrix $U_{\lambda_{1}} \cdots U_{\lambda_{n}}$ $i s^{-d e f i n e d . ~ L e t ~} R$ be the polynomial ring over $K$ generated by the entries of all the $U_{\lambda}$, and let $V$ be the $K$-vector subspace of $R$ spanned by all the entries of all the product matrices $U_{\lambda_{1}} \cdots U_{\lambda_{n}}$ of the form just described. Then $V$ is linearly radical.

The author does not know whether (a) holds for arbitrary $r \leqq s$. If it did, it would give a new proof in characteristic zero of the fact that certain ideals $\mathfrak{A}(B)$ described in [3] are radical, while (b) gives another proof in characteristic zero that the ideals $I_{H, n}$ described in [4] and [5] are radical. In each case, the fact that the ideals in the class are radical is the key point in the proof that certain rings of invariants are Cohen-Macaulay. This is discussed further in $\S 4$.

2. Proof of Theorem 1. We need some preliminary observations and results. Let $S$ be a Noetherian graded $K$-algebra and $M$ the irrelevant maximal ideal. Then we can always choose a system of parameters for $S$ consisting of forms, i.e. if $\operatorname{dim} S=d$, we can choose forms $f_{1}, \cdots, f_{d}$ in $M$ such that the ideal they generate is primary to $M$, in which case $S$ is a finitely generated graded module over the polynomial subring $K\left[f_{1}, \cdots, f_{d}\right]$. Moreover, if graded $M=g$ (the grade of a proper ideal is the length of the longest $R$-sequence contained in it), then there is an $R$-sequence of forms in $M$ of length $g$. These assertions follow by standard arguments from the easy observation:

(2.1) If $S$ is a Noetherian graded $K$-algebra, $I$ is a homogeneous ideal of $S$, and $P_{1}, \cdots, P_{n}$ are primes of $S$ such that for each $i, I \not \subset$ $P_{i}$, then there is a form $f$ in $I$ such that $f \notin \bigcup_{i} P_{i}$.

We note that by the analogue in the graded case of Theorem (25.16) of [7] that $S$ is Cohen-Macaulay if and only if for every system of parameters consisting of forms $f_{1}, \cdots, f_{d}, S$ is a finitely generated free graded module over the polynomial ring $K\left[f_{1}, \cdots, f_{d}\right]$. It follows that if $S$ is Cohen-Macaulay and $f_{1}, \cdots, f_{k}$ is an $R$-sequence of forms in 
$M$, then $S$ is free over $K\left[f_{1}, \cdots, f_{k}\right]$, for $f_{1}, \cdots, f_{k}$ can be extended to an $R$-sequence of length $d$ consisting of forms, and $K\left[f_{1}, \cdots, f_{d}\right]$ is free over $K\left[f_{1}, \cdots, f_{k}\right]$.

We next observe:

(2.2) If $S$ is free $R$-algebra (or merely a free $R$-module), then for any family of ideals $\left\{I_{\lambda}: \lambda \in \Lambda\right\}$ in $R,\left(\bigcap_{\lambda} I_{\lambda}\right) S=\bigcap_{\lambda}\left(I_{\lambda} S\right)$.

(2.3) If $S$ is a graded Noetherian $K$-algebra, where $K$ is a field, and $R$ is a subring of $S$ generated over $K$ by a finite number of forms $f_{1}, \cdots, f_{m}$ of positive degree such that $S$ is $R$-fiat, then $S$ is free $R$ module. In fact, if $\left\{s_{j}: j\right\}$ is a family of forms in $S$ such that $\left\{s_{j}^{\prime}\right\}$ is a $K$-vector space basis for $S / P S$, where $P=\left(f_{1}, \cdots, f_{m}\right)$ and 'denotes reduction modulo $P$, then $\left\{s_{j}: j\right\}$ is a free basis for $S$ over $R$. (We can always choose one of the $s_{j}$ to be 1 , if we wish.)

Proof. We use Noetherian induction on the homogeneous ideals of $R$. If $P=(0)$ then $R=K$ and there is nothing to prove. Otherwise there is a nonzero form $f$ in $P$ such that either (1) $f$ is not a zerodivisor in $R$ or (2) $f P=(0)$, according as grade $P>0$ or grade $P=0$. In either case, if $*$ denotes reduction modulo $(f)$, we have that $\left\{s_{j}^{*}: j\right\}$ is a free basis for $S / f S$ ever $R / f R$, by the induction hypothesis. ( $S / f S$ is flat over $R / f R$ by (18.10) of [7].) The $\left\{s_{j}: j\right\}$ span $S$ as an $R$-module, for if $g$ were a form of least degree in $S-\sum_{j} R s_{j}$, we would have $g=\sum_{j} r_{j} s_{j}+\sum_{i} f_{i} t_{i}$, where each $r_{j} \in R$, for each $j$, deg $r_{j}+$ $\operatorname{deg} s_{j}=\operatorname{deg} g$, and for each $i, \operatorname{deg} f_{i}+\operatorname{deg} t_{i}=\operatorname{deg} g$. Since the $f_{i}$ have positive degree, each $t_{i} \in \sum_{j} R s_{j}$, and since each $f_{i} \in R, g \in \sum_{j} R s_{j}$.

Now suppose that there is a nonzero relation $\sum_{i} r_{j} s_{j}=0$, where the $r_{j} \in R$. We can choose such a relation in which the $r_{j}$ are forms and $\max _{j} \operatorname{deg} r_{j}$ is a small as possible. Since $\sum_{j} r_{j}^{*} s_{j}^{*}=0$, each $r_{j} \in$ $(f)$, and we have $r_{j}=f t_{j}$, where $t_{j} \in R$, for each $j$. In case (1), since $f$ is not a zerodivisor in $R$ and $S$ is $R$-flat, $f$ is not a zerodivisor in $S$, and $\sum_{j} t_{j} s_{j}=0$, contradicting the minimality of $\max _{j} \operatorname{deg} r_{j}$. In case (2) $\sum_{j} t_{j} s_{j} \in \mathrm{Ann}_{s} f=\left(\mathrm{Ann}_{R} f\right) S$ (since $S$ is $R$-flat) $=P S$, so that $\sum_{j} t_{j}^{\prime} s_{j}^{\prime}=0$. Hence, each $t_{j} \in P$, and each $r_{j}=f t_{j}=0$.

(2.4) With the same hypothesis and notation as (2.3), if $S / P$ is Cohen-Macaulay and $g_{1}, \cdots, g_{t}$ are forms of $S$ such that $g_{1}^{\prime}, \cdots, g_{t}^{\prime}$ is a system of parameters for $S / P$, then $g_{1}, \cdots, g_{t}$ are algebraically independent over $R$, and for each $q, 0 \leqq q \leqq t, S$ is free over $R\left[g_{1}, \cdots, g_{q}\right]$.

Proof. Since $S / P$ is Cohen-Macaulay, it is a finitely generated graded free module over $K\left[g_{1}^{\prime}, \cdots, g_{t}^{\prime}\right]$. Let $h_{1}, \cdots, h_{k}$ be forms of $S$ such that $h_{1}^{\prime}, \cdots, h_{k}^{\prime}$ is a free basis for $S / P$ over $K\left[g_{1}^{\prime}, \cdots, g_{t}^{\prime}\right]$. We 
can assume that $h_{1}=1$. Then it is clear that the products $h_{i}^{\prime} M^{\prime}$, where $M$ is monomial in $g_{1}, \cdots, g_{t}$, form a $K$-vector space basis for $S / P$ over $K$. By (2.3), the products $h_{i} M$ are a free basis for $S$ over $R$, and the result is then clear.

(2.5) If $T$ is a Cohen-Macaulay graded Noetherian $K$-algebra and $I$ is a homogeneous ideal of $T$ such that $T / I$ is Cohen-Macaulay, then for every ideal $J$ of $T$ containing $I$, grade $J=$ gade $J / I+$ grade $I$.

Proof. Since $T$ and $T / I$ are graded and Cohen-Macaulay, all minimal primes of $I$ have the same grade $g=$ grade $I$. It suffices to consider the case where $J$ is a prime which contains $I$, and localizing at $J$ will not change the grades of $J, I$ or $J / I$. Hence, we may assume instead that $T$ is a Cohen-Macaulay local ring with maximal ideal $J$, and that $T / I$ is Cohen-Macaulay. Let $P$ be any minimal prime of $I$. Then $\operatorname{grade} I=\operatorname{rank} P, \operatorname{grade} J / I=\operatorname{rank} J / I=\operatorname{rank} J / P, \operatorname{grade} J=$ rank $J$, and the result follows.

We are now ready to give the proof of Theorem 1. By replacing $R, S$ by $L \otimes_{K} R, L \otimes_{K} S$, where $L$ is an algebraic closure of $K$, we may assume without loss of generality that $K$ is algebraically closed. By (2.3) $S$ is faithfully flat (even free) over $R$, and since $S$ is CohenMacaulay we can conclude that $R$ is Cohen-Macaulay and that for each maximal ideal $Q$ of $R$ that $S / Q S$ is Cohen-Macaulay.

We know that every radical ideal of $R$ is an intersection of maximal ideals of $R$, and it follows from (2.2) and (2.3) that it is enough to show that for each maximal ideal $Q$ of $R, Q S$ is radical in $S$. To show that $Q S$ is radical it is necessary and sufficient to prove that there is an element $f$ in a defining ideal for the singular locus of the variety defined by $Q S$ such that $f$ is not a zerodivisor on $Q S$.

Let us represent $S$ as a quotient of a polynomial ring $T=$ $K\left[x_{1}, \cdots, x_{t}\right]$ by a homogeneous ideal $I$. (The $x_{i}$ need not have degree one.) Let $F_{1}, \cdots, F_{m}$ be forms in $T$ whose residues modulo $I$ are $f_{1}, \cdots, f_{m}$, and let us denote a set of forms which generate $I$ by $F_{m+1}, \cdots, F_{r}$. Thus, $T /\left(F_{1}, \cdots, F_{r}\right) \cong S / P$, where $P=\left(f_{1}, \cdots, f_{m}\right)$.

Any maximal ideal $Q$ of $R$ has the form $\left(f_{1}-c_{1}, \cdots, f_{m}-c_{m}\right)$ for suitable choices of $c_{1}, \cdots, c_{m}$ in $K$. When $Q=P, c_{1}=\cdots=c_{m}=0$.

We claim that for any associated prime $Q_{1}$ of $Q S$ in $S$, rank $Q_{1}=$ $d$, where $d=\operatorname{dim} R$. In fact, since $S / Q S$ is Cohen-Macaulay, every associated prime $Q_{1}$ of $Q S$ is minimal, and since $S$ is flat over $R$, $Q_{1} \cap R=Q$. Then $R_{Q} \rightarrow S_{Q_{1}}$ is a faithfully flat local extension, and the fiber $S_{Q_{1}} / P S_{Q_{1}}$ has dimension zero, since $Q_{1}$ is minimal for $P S$. Hence, $\operatorname{rank} Q_{1}=\operatorname{dim} S_{Q_{1}}=\operatorname{dim} R_{Q}+\operatorname{dim} S_{Q_{1}} / P S_{Q_{1}}$ (see [2], Corollaire 
6.1.2, p. 135) $=\operatorname{dim} R_{Q}=\operatorname{rank} Q=\operatorname{dim} R$, for $R$ is homogeneous and Cohen-Macaulay and all maximal ideals therefore have the same rank.

The associated primes of $\left(F_{1}-c_{1}, \cdots, F_{m}-c_{m}, F_{m+1}, \cdots, F_{r}\right)=Q^{*}$ in $T$ are in one-to-one correspondence with those of $Q S$ in $S$, in an obvious way, so that if $g$ denotes the grade of $I$, then by (2.5) each associated prime $H$ of $Q^{*}$ has grade (= rank) $g+d$. It follows that a defining ideal for the singular locus of the variety defined by $Q^{*}$ is $Q^{*}+I_{g+d}\left(\partial F_{i} / \partial x_{j}\right)$, where $I_{g+d}\left(\partial F_{i} / \partial x_{j}\right)$ is the ideal generated by the $g+d$ by $g+d$ minors of the $r$ by $t$ Jacobian matrix obtained from $F_{1}-c_{1}, \cdots, F_{m}-c_{m}, F_{m+1}, \cdots, F_{r}$ (the constants drop out after differentiation). See [7], (46.3) and (39.11).

In the case where $Q=P$, we know that $P^{*}$ is radical, and it follows from (2.1) that there is a form $F$ in $P^{*}+I_{g+d}\left(\partial F_{i} / \partial x_{j}\right)$ which is not a zerodivisor on $P^{*}$. Clearly, we can choose such an $F$ in $I_{g+d}\left(\partial F_{i} / \partial x_{j}\right)$, and we then have that the image $f$ of $F$ in $S$ is not a zerodivisor on PS. By (2.4), $S$ is a free module over $R[f]$, and $f$ is an indeterminate over $R$.

Now let $Q$ be any maximal ideal of $R$. Then $F \in I_{g+d}\left(\partial F_{i} / \partial x_{j}\right) \subset$ $Q^{*}+I_{g+d}\left(\partial F_{i} / \partial x_{j}\right)$, and to complete the proof it suffices to show that $f$ is not a zerodivisor on $Q S$. Since $S$ is free over $R[f]$, it is enough to show that $f$ is not a zerodivisor on $Q R[f]$. Since $Q$ is an ideal of $R$ and $f$ is an indeterminate over $R$, this is clear.

3. Proof of Theorem 2. We first restate Propositions 21 and 22 of [5].

(3.1) Let $R$ be a Noetherian ring and let $x_{1}, \cdots, x_{n}$ be elements of $R$ which generate an ideal of grade $g$. Let $t_{i j}, 1 \leqq i \leqq g, 1 \leqq j \leqq n$, be $g n$ new indeterminates. Then the elements $\sum_{j} t_{i j} x_{i}$ form an $R$ sequence in $R\left[t_{i j}: i, j\right]$ and if $R$ is a domain (respectively, reduced) and $i \leqq g-1$ then the ideal generated by the first $i$ terms is prime (respectively, radical).

We can now establish the lemma. It is clear that if $I$ is semiregular, then the ideals in question are radical, for the ideal $M / I$ will have rank and hence grade $d$ in the reduced Cohen-Macaulay ring $R / I$, and hence the power associated ideal generated by the images of $x_{1}, \cdots, x_{n}$ will also have grade $d$.

To prove the converse, suppose that the ideals described are radical. To show that $R / I$ is Cohen-Macaulay, it suffices to show that $(R / I)_{M / I}$ is Cohen-Macaulay (see, for example, Proposition 19 of [5]). It is then sufficient to prove the following local result: 
(3.2) Let $(R, M)$ be a reduced local ring of dimension $d$, and let $x_{1}, \cdots, x_{n}$ be elements of $M$ which generate an ideal whose radical is $M$. Let $t_{i j}$ be $(d-1) n$ new indeterminates over $R$, as before. Then $R$ is Cohen-Macaulay if and only if each of the ideals $\left(\sum_{j} t_{1 j} x_{j}, \cdots, \sum_{j} t_{i j} x_{j}\right)$, $1 \leqq i \leqq d-1$, is radical in $R\left[t_{i j}: i, j\right]$.

Proof. The necessity of the condition is clear from (3.1). Now suppose that the ideals are radical. If $d=0$ there is nothing to prove. If $d>0$, then since (0) is radical $M$ cannot be a minimal prime of (0) and hence cannot be an associated prime of (0), and the grade of $M$ is $\geqq 1$.

We use induction on $d$. If $d=1$, we are done. If $d>1$, then we know that $\sum_{j} t_{1 j} x_{j}$ is not a zerodivisor in $R\left[t_{1 j}: j\right]$, and hence also not a zerodivisor in $R\left(t_{1 j}: j\right)=R\left[t_{1 j}: j\right]_{M^{\prime}}$, where $M^{\prime}$ is the extension of $M$ to $R\left[t_{1 j}: j\right] . \quad R\left(t_{1 i}: j\right)$ is a faithfully flat local extension of $R$, and $R\left(t_{1 j}: j\right) / M=K\left(t_{1 j}: j\right)$. It suffices to show that $R\left(t_{1 j}: j\right)$ is CohenMacaulay. But we may now apply the induction hypothesis to $R\left(t_{1 j}: j\right) /\left(\sum_{j} t_{1 j} x_{j}\right)$.

We are now ready to prove Theorem 2. Let $d=\operatorname{dim} K[x] / I$. If $d=n, I=(0)$ and we are done. Assume that $d \leqq n-1$, so that $d-1 \leqq n-2$. Then it suffices to show that if $1 \leqq p \leqq n-2$, and $T=\left(t_{i j}\right)$ is a $p$ by $n$ matrix of new indeterminates, then $I+$ $\left(\sum_{j} t_{1 j} x_{j}, \cdots, \sum_{j} t_{p j} x_{j}\right)$ is a radical ideal in $K[x, t]$. Hence, it suffices to show that the image of $I$ in $R_{1}=K[x, t] /\left(\sum_{j} t_{1 j} x_{j}, \cdots, \sum_{j} t_{p j} x_{j}\right)$ is radical. Let $Y=\left(y_{i j}\right)$ be the matrix of indeterminates described in Theorem 2 , and let $Z=\left(z_{i j}\right)$ be a $p$ by $n-1$ matrix of new indeterminates.

We have a $K$-homomorphism $\phi$ from $K[x, t]$ to $K[y, z]$ which takes each $x_{i}$ to $\Delta_{i}$, and which takes each entry of $T$ to the corresponding entry of the product matrix $Z Y$. Let $P=\operatorname{ker} \phi$. We shall show that $P=P^{\prime}$, where $P^{\prime}=\left(\sum_{j} t_{1 j} x_{j}, \cdots, \sum_{j} t_{p j} x_{j}\right)$. It is clear that $P^{\prime} \subset P$, and $P^{\prime}$ is prime by (3.1). It is then easy to see that $P^{\prime}=P$ : the point is that given $n-2$ or fewer vectors in $L^{n}$, where $L$ is any field, and a nonzero (respectively, a zero) vector $v$ orthogonal to all of them, then we can choose $n-1$ independent (respectively, dependent) vectors in the orthogonal complement of $v$ such that (1) the original $n-2$ or fewer vectors are in their span, and (2) if they form the rows of a matrix, the $n-1$ by $n-1$ minors of that matrix are the components of $v$.

Hence, we may regard $K[x, t] / P$ as a subring of $K[y, z]$. Let $I^{*}$ be the image of $I$ in $K[x, t] / P$. Our hypothesis gives us that $I^{*} K[y, z]$ is radical: the presence of the $z$ 's is irrelevant, since they do not occur in the generators for $I^{*}$. 
Let $R_{2}$ be the subring of $K[y, z]$ generated by the $\Delta_{i}$ and the entries of the product matrix $Z Y$, so that $R_{2}=\operatorname{Im} \phi$ and $R_{1} \cong R_{2}$. It suffices to show that $I^{*} K[y, z] \cap R_{2}=I^{*} R_{2}$. It is actually the case that if $J$ is any ideal of $R_{2}$ then $J K[y, z] \cap R_{2}=J$.

To see this, let $S L(n-1, K)$ act on the 1-forms of $K[y, z]$ thus: if $C \in S L(n-1, K)$, let $C$ act by taking the entries of $Y$ (respectively, $Z$ ) into the corresponding ones of $C Y$ (respectively, $Z C^{-1}$ ). This extends to a rational action of $S L(n-1, K)$ on $K[y, z]$. The ring of invariants is known classically to be precisely $R_{2}$. (See [8], Theorem (2.6.A), p. 45.) Note that if we had $p \geqq n-1$, the reasoning would fail, for the $n-1$ by $n-1$ minors of $Z$ would also be invariant.) Since $S L(n-1, K)$ is reductive, we have a Reynolds retraction $K[y, z] \rightarrow R_{2}$ (which is a map of $R_{2}$-modules), and it follows that every ideal of $R_{2}$ when expanded to $K[y, z]$ and then contracted back to $R_{2}$ contracts to itself. (See [1], $\S \mathrm{V}-2$, [5], Propositions 9 and 10.)

4. Linearly radical spaces. Throughout this section, $K$ is a field, $R$ is a $K$-algebra, and $V$ is a $K$-vector subspace of $R$. We first note two trivial facts:

(4.1) If $V$ is linearly radical in $R$, and $I=\left(f_{1}, \cdots, f_{m}\right)$, where $f_{1}, \cdots, f_{m} \in V$, then $V / V \cap I$ is linearly radical in $R / I$.

(4.2) If $V$ is linearly radical in $R$ and $x_{1}, \cdots, x_{n}$ are indeterminates over $R$, then $V+\sum_{i} K x_{i}$ is linearly radical in $R\left[x_{1}, \cdots, x_{n}\right]$.

Note that if $V \subset R$ is linearly radical, then $R$ is reduced, for $0 \in V$, and (0) must be radical.

The next result is the crucial point in the proof of Theorem $3 \mathrm{~b}$ ).

(4.3) If $V$ is linearly radical in $R, x$ is an indeterminate over $R$, and $f \in V$, then the subspace of $R[x]$ spanned by $V$ and $x f$ is linearly radical.

Proof. Each finite-dimensional subspace of $V+K x f$ has a basis consisting of at most one element of the form $g+c x f, g \in V, c \in K$, and several elements $v_{1}, \cdots, v_{k}$ of $V$. We may assume that we have already divided out by $\left(v_{1}, \cdots, v_{k}\right)$. We may also assume that $K$ is algebraically closed. It then suffices to show that if $f, g \in V$, then $(f x+g)$ is radical in $R[x]$.

Let $F \in R[x]$ be an element of the radical of $(f x+g)$ not in $(f x+g)$. If we localize at $K[x]-\{0\}$, the resulting ring is $K(x) \otimes_{K} R$, and then $f x+g \in K(x) \bigotimes_{K} V$, and so generates a radical ideal. It follows that there is a nonconstant polynomial $p$ in $K[x]-\{0\}$ such 
that $p F \in(f x+g)$. We can write $p=\left(x-c_{1}\right) \cdots\left(x-c_{m}\right)$, where $c_{1}, \cdots, c_{m} \in K$. Let $p_{t}=\left(x-c_{1}\right) \cdots\left(x-c_{t}\right), 0 \leqq t \leqq m\left(p_{0}=1\right)$. Then for some $t, 0 \leqq t \leqq m-1$. $p_{t} F \notin(f x+g)$ while $\left(x-c_{t+1}\right) p_{t} F \in(f x+g)$. Replacing $F$ by $p_{t} F$, we can assume that $F \notin(f x+g)$ but $(x-c) F \in$ $(f x+g)$ for a certain $c \in K$. After a change of variable $x-c \mapsto x$, we can assume that $x F \in(f x+g)$, but $F \notin(f x+g)$ (of course, $F$ is altered by the change of variable, and we also have a new $g: f x+g=$ $f(x-c)+(g+c f))$. After replacing $F$ by a suitable power, we can also assume that $F \notin(f x+g)$ but $F^{2} \in(f x+g)$.

Let us write $F=x F_{1}+r_{0}$, where $r_{0} \in R$ is the constant term of $F$. Since $x F, F^{2} \in(f x+g)$, we have that $r_{0} F \in(f x+g)$. We can also write:

$$
x F=\left(G x^{2}+s_{1} x+s_{0}\right)(f x+g),
$$

where $s_{0}, s_{1} \in R$. Equating like coefficients, we have that $s_{0} g=0$, and that $r_{0}=s_{1} g+s_{0} f$. We can also write $r_{0} F=\left(H x+t_{0}\right)(f x+g)$, where $t_{0} \in R$, and equating constant terms gives that $r_{0}^{2}=t_{0} g$. Then:

$$
\begin{aligned}
\left(s_{0} f\right)^{3} & =\left(r_{0}-s_{1} g\right)^{2}\left(s_{0} f\right)=r_{2}^{0}\left(s_{0} f\right)-\left(s_{0} g\right) f\left(2 r_{0} s_{1}+s_{1}^{2} g\right) \\
& =\left(t_{0} g\right)\left(s_{0} f\right)-0\left(\text { since } r_{0}^{2}=t_{0} g \text { and } s_{0} g=0\right) \\
& =0
\end{aligned}
$$

Hence, $s_{0} f=0$, since $R$ is reduced. But then $\left(^{*}\right)$ yields $x F=$ $\left(G x^{2}+s_{1} x\right)(f x+g)$, or $F=\left(G x+s_{1}\right)(f x+g) \in(f x+g)$, as required.

From (4.3) we can deduce at once:

(4.4) If $V$ is a linearly radical subspace of $R, f_{1}, \cdots, f_{m} \in V$, and $U=\left(u_{i j}\right)$ is an $m$ by $n$ matrix of inderminates over $R$, then the subspace $W$ of $R\left[u_{i j}: i, j\right]$ spanned by $V$ and the entries of the product matrix $\left(f_{1} \cdots f_{m}\right) U$ is linearly radical. (Note that (4.3) is the case $m=n=1$.)

Proof. By repeated applications of (4.3), the space $V^{\prime}$ spanned by $V$ and the elements $f_{i} u_{i j}$ is linearly radical, and $W$ is a subspace of $V^{\prime}$.

The following result contains Theorem $3 b)$.

(4.5) Let $\Lambda$ be a partially ordered set as in Theorem 3b) and suppose that for each minimal element $\lambda \in \Lambda, U_{\lambda}$ is a one-rowed matrix with entries in a linearly radical subspace $V$ or $R$, while for the set of nonminimal $\lambda$ the $U_{\lambda}$ are matrices of indeterminates with all entries distinct. Then the space spanned by $V$ and the entries of the product matrices $U_{\lambda_{1}} \cdots U_{\lambda_{n}}$ of the same type described in 
Theorem 3b) (let us call these the admissible products) is linearly radical.

Proof. Clearly, we may reduce to the case where $\Lambda$ is finite. Let $\Lambda_{1}$ be the set of minimal elements of $\Lambda$, let $\Lambda^{\prime}=\Lambda-\Lambda_{1}$, and let $\Lambda_{2}$ be the set of minimal elements of $\Lambda^{\prime}$. Let $V^{\prime}$ be the space spanned by $V$ and the entries of the admissible products $U_{\lambda_{1}} U_{\lambda_{2}}$, where, necessarily, $\lambda_{i} \in \Lambda_{i}, i=1,2$, and $\lambda_{1}<\lambda_{2}$. By repeated applications of (4.4), $V^{\prime}$ is linearly radical.

If we replace $V, \Delta,\left\{U_{\lambda}: \lambda \in \Lambda\right\}$ by $V^{\prime}, \Lambda^{\prime},\left\{U_{\lambda}^{\prime}: \lambda \in \Lambda^{\prime}\right\}$, where $U_{\lambda}^{\prime}=$ $U_{\lambda}$ if $\lambda \in \Lambda^{\prime}-\Lambda_{2}$, while $U_{\lambda}=U_{\lambda_{1}} U_{\lambda_{2}}$ if $\lambda=\lambda_{2} \in \Lambda_{2}$ (and $\lambda_{1}$ is the unique predecessor of $\lambda_{2}$ ), then $V^{\prime}, \Lambda^{\prime}$, and $\left\{U_{\lambda}^{\prime}: \lambda \in \Lambda^{\prime}\right\}$ again satisfy our hypotheses, and the result follows by induction on the length of the longest chain in $\Lambda$.

Consider now the situation of Theorem $3 \mathrm{~b}$ ) with $\Lambda$ finite. The entries of the admissible products $U_{\lambda_{1}} \cdots U_{\lambda_{n}}$ such that $\lambda_{n}$ is maximal can be shown to generate the ring of invariants of an action of a product of general linear groups on $R$, at least if $K$ has characteristic zero. It was facts of this kind which led the author to prove Theorem $3 b)$.

Suppose that $K$ has characteristic zero and consider the class of ideals $I_{H, n}$ discussed in [4] and [5]. Working from Propositions 25, 26,27 , and 28 of [5], we have an embedding $K[X] / I_{H} \rightarrow K[u, v]$, such that the image $K\left[U^{*} V\right]$ is the ring of invariants of a reductive linear algebraic group acting on $K[u, v]$. Just as in the last paragraph of the proof of Theorem 2, there is a Reynolds operator from $K[u, v]$ to $K\left[U^{*} V\right]$, and to show that $I_{H, n}$ is radical it suffices to show that $I_{H, n} / I_{H}$ expands to a radical ideal in $K[u, v]$. But $I_{H, n} / I_{H}$, expanded to $K[u, v]$, is generated by a subset of the first row of the matrix $U^{*} V$, and this first row spans a linearly radical space, by Theorem $3 b)$.

As was mentioned before, proving that the ideals $I_{H, n}$ are radical is critical in proving that they are also semiregular.

The situation with respect to the result of Theorem 3a) is quite similar. Suppose we knew that the space spanned by the minors is linearly radical for all $r \leqq s$, or even the weaker result that any subset of the minors generates a radical ideal (which we prove below for $r=2$ ). A Reynolds operator argument then shows that within the homogeneous coordinate ring of the Grassmannian of $r$-dimensional subspaces of $K^{r}$, the ideal generated by a subset of the $r$ by $r$ minors is radical (this homogeneous coordinate ring is generated as a $K$ algebra by the $r$ by $r$ minors of an $r$ by $s$ matrix of indeterminates over $K$ ) when $K$ has characteristic zero. The point is that the $r$ by $r$ 
minors generate the ring of invariants of an action of $S L(r, K)$ on the polynomial ring generated over $K$ by the entries of the $r$ by $s$ matrix of indeterminates. In particular, the ideals $\mathfrak{A}(B), B \in \mathscr{B}$, and in particular, the ideals $\mathfrak{U}\left(B_{o}\right)$, discussed in [3], are radical, and this is a critical point in showing that homogeneous coordinate rings of Grassmann varieties and their Schubert subvarieties are Cohen-Macaulay.

We now prove Theorem 3a) for the case $s=r+1$. It is easy to see that the linear changes of coordinates of the $y_{i j}$ which are uniform on the colums of $Y=\left(y_{i j}\right)$ are in one-to-one correspondence with the linear changes of coordinates in the vectors space spanned by the $\Delta_{i}$. From this it follows that we need only prove:

(4.6) Let $Y=\left(y_{i j}\right)$ be an $r$ by $r+1$ matrix of indeterminates over a field $K$. Let $J_{t}$ be the ideal $\left(\Delta_{i}: 1 \leqq i \leqq r+1-t\right)$, where $\Delta_{i}$ is the $r$ by $r$ minor obtained by deleting the $i$ th column. Then $J_{t}$ is radical for each $t$.

Proof. If $t=0, J_{t}$ is known to be prime (see, for example, Proposition 26 of [5]) while the result is clear is $t \geqq r$. Hence, we assume that $r>t>0$, and we use induction on $t$, i.e. we assume the result for $t^{\prime}<t$. It is easy to see that $\sqrt{J_{t}}=J_{0} \cap I_{t}$, where $I_{t}$ is the ideal generated by the $t$ by $t$ minors of the matrix formed from the last $t$ columns of $Y$, and is therefore prime. In fact, since we are working with radical ideals, we need only see a corresponding fact about unions of varieties, and the result reduces to the observation that given an $r$ by $r+1$ matrix with entries in a field, then the $r$ by $r$ minors which contain the last $t$ columns all vanish if and only if either all the $r$ by $r$ minors vanish (i.e. the matrix has rank $\leqq r-1$ ) or else the $t$ by $t$ minors of the last $t$ columns vanish (the last $t$ columns are dependent). For if the last $t$ columns are independent and the matrix has rank $r$, we can find $r$ independent columns containing the last $t$, and they give rise to a nonvanishing minor. Now $J_{t-1}=J_{t}+(\Delta)$ is radical, where $\Delta=\Delta_{r-t+2}$, by the induction hypothesis, so that $\sqrt{J_{t}} \subset J_{t}+(\Delta)$, and it follows that $\sqrt{J_{t}} \subset J_{t}+$

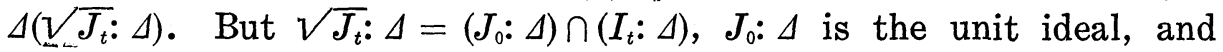
$I_{t}$ is a prime not containing $\Delta$, so that $I_{t}: \Delta=I_{t}$. Thus, $\sqrt{J_{t}}=J_{t}+\Delta I_{t}$. It suffices to show that if $A$ is any $t$ by $t$ minor of the last $t$ columns of $Y$, then $A \Delta \in J_{t}$. We might as well consider the case where $A$ is formed from the first $t$ rows. Now $Y$ can be decomposed into submatrices thus:

$$
Y=\left(\begin{array}{lll}
Y_{1} & Y_{2} & Y_{3} \\
Z_{1} & Z_{2} & Z_{3}
\end{array}\right)
$$


where the $Y_{i}$ have $t$ rows, the $Z_{i}$ have $r-t$ rows, and $Y_{i}$ and $Z_{i}$ both have either $r+1-t, 1$, or $t-1$ columns, according as $i=1$, 2, or 3. Thus

$$
\Delta=\operatorname{det}\left(\begin{array}{ll}
Y_{1} & Y_{3} \\
Z_{1} & Z_{3}
\end{array}\right)
$$

and $A=\operatorname{det}\left(\begin{array}{ll}Y_{2} & Y_{3}\end{array}\right)$. Form an auxiliary $r+t$ by $r+t$ matrix

$$
Y^{*}=\left(\begin{array}{llll}
Y_{1} & Y_{2} & Y_{3} & 0 \\
Z_{1} & Z_{2} & Z_{3} & 0 \\
0 & Y_{2} & Y_{3} & Y_{3}
\end{array}\right) \text {. }
$$

Then $\operatorname{det} Y^{*}= \pm A \Delta$. To see this, subtract each of the last $t-1$ columns from the corresponding one of the next to last $t-1$ columns. After permuting the columns, we have that $\operatorname{det} Y^{*}$ is the same, up to sign, as the determinant of

$$
\left(\begin{array}{llll}
Y_{1} & Y_{3} & Y_{2} & 0 \\
Z_{1} & Z_{3} & Z_{2} & 0 \\
0 & 0 & Y_{2} & Y_{3}
\end{array}\right) .
$$

This matrix has block form, and the determinant is clearly $A \Delta$. On the other hand, if we subtract each of the first $t$ rows of $Y^{*}$ from the corresponding one of the last $t$, we find that $Y^{*}$ has the same determinant as

$$
\left(\begin{array}{clll}
Y_{1} & Y_{2} & Y_{3} & 0 \\
Z_{1} & Z_{2} & Z_{3} & 0 \\
-Y_{1} & 0 & 0 & Y_{3}
\end{array}\right) .
$$

Any nonzero $r$ by $r$ minor of the first $r$ rows comes from $Y$, and if that minor does not contain the last $t$ columns of $Y$, then its cofactor has a column which is 0 . Hence, the determinant of this last matrix is in $J_{t}$, and $A \Delta \in J_{t}$ as required.

We need the following result before we can prove the rest of Theorem 3a).

(4.7) Let $s$ be an integer such that if $x_{1}, \cdots, x_{s}$ and $y_{1}, \cdots, y_{s}$ are indeterminates over a field $K$, then any subset of the 2 by 2 minors of

$$
\left(\begin{array}{l}
x_{1} \cdots x_{s} \\
y_{1} \cdots y_{s}
\end{array}\right)
$$


generates a radical ideal in $R=K\left[x_{1}, y_{1}, \cdots, x_{s}, y_{s}\right]$. Then for every field $K$, for every $c \in K$, and for every nonnegative integer $n$, if $x_{1}, \cdots, x_{s}, y_{1}, \cdots, y_{s}$, and $u_{1}, \cdots, u_{n}$ are independent indeterminates over $K$, then any subset of the 2 by 2 minors of

$$
\left(\begin{array}{lll}
u_{1} \cdots u_{n} & x_{1} \cdots x_{s} \\
c u_{1} \cdots c u_{n} & y_{1} \cdots y_{s}
\end{array}\right)
$$

generates a radical ideal in $R[u]=R\left[u_{1}, \cdots, u_{n}\right]$.

Proof. We use induction on $s$. Notice that if $s$ has the required property so does each $s^{\prime}<s$, and we may assume that if a matrix has the required form with $s^{\prime}<s$ and any $n$, then any subset of its 2 by 2 minors generates a radical ideal. We then use induction on $n$. First note that by subtracting $c$ times the first row from the second we may assume that $c=0$ (there is a linear change of coordinates on the $x$ 's and $y$ 's). Let $J$ be any ideal generated by a subset of the 2 by 2 minors. Let $I$ be the subideal of $J$ generated by those of the minors which do not involve the column which contains $u_{n}$. By the induction hypothesis, $I$ is radical, and $J$ is generated over $I$ by certain of the products $u_{n} y_{i}$. After renumbering, we can assume that $J=I+u_{n}\left(y_{1}, \cdots, y_{k}\right), 1 \leqq k \leqq s$ (if $J=I$ we are done). We claim that $J=\left(I+\left(u_{n}\right)\right) \cap\left(I+\left(y_{1}, \cdots, y_{k}\right)\right)$. Call the intersection $J^{\prime}$, and suppose that $j=i+u_{n} t \in J^{\prime}$, where $i \in I$. Then $u_{n} t \in I+\left(y_{1}, \cdots, y_{k}\right)$. $u_{n}$ is not a zerodivisor on $I+\left(y_{1}, \cdots, y_{k}\right)$, since the generators of that ideal do not involve $u_{n}$. Hence, $t \in I+\left(y_{1}, \cdots, y_{k}\right)$, and our claim is established.

It suffices then to show that $I+\left(u_{n}\right)$ and $I+\left(y_{1}, \cdots, y_{k}\right)$ are radical. The first is clear, since $I$ is radical and its generators do not involve $u_{n}$. On the other hand, $R[u] /\left(I+\left(y_{1}, \cdots, y_{k}\right)\right)$ is isomorphic to a quotient of $K\left[x_{k+1}, y_{k+1}, \cdots, x_{s}, y_{s}\right]\left[u_{1}, \cdots, u_{n}, x_{1}, \cdots, x_{k}\right]$ by an ideal generated by a subset of the 2 by 2 minors of

$$
\left(\begin{array}{llll}
u_{1} \cdots u_{n} & x_{1} \cdots x_{k} & x_{k+1} \cdots x_{s} \\
0 \cdots 0 & 0 \cdots 0 & y_{k+1} \cdots y_{s}
\end{array}\right)
$$

and hence is reduced by a special case of the result for $s^{\prime}=s-k<s$.

We can now prove the last statement of Theorem (3a). We use induction on $s$. We must show that an ideal $J$ generated by a subset of the minors of

$$
\left(\begin{array}{llll}
x & x_{1} & \cdots & x_{s} \\
y & y_{1} & \cdots & y_{s}
\end{array}\right)
$$

is radical, given the result for smaller such matrices of indeterminates. 
Let $I$ be the subideal of $J$ generated by those minors which do not involve the first column. $I$ is radical by the induction hypothesis. After a renumbering we can assume that $J=I+J_{1}$, where $J_{1}$ is the ideal generated by those 2 by 2 minors which have first column equal to $x_{y}^{x}$ and second column equal to ${ }_{y_{i}}^{x_{i}}$ for some $i, 1 \leqq i \leqq k$, where $k$ is a fixed integer such that $1 \leqq k \leqq s$ (if $J=I$ we are done). Let $H$ be the ideal generated by the 2 by 2 minors of

$$
\left(\begin{array}{l}
x_{1} \cdots x_{k} \\
y_{1} \cdots y_{k}
\end{array}\right)
$$

We first show that $J=(I+(x, y)) \cap(J+H)$. Let $J^{\prime}$ denote the intersection. Suppose that $j^{\prime}=j+h \in J^{\prime}$, where $j \in J$ and $h \in H$. Then $h \in J^{\prime}$ and it suffices to show that $h \in J$. But $h \in(H+I) \cap((x, y)+I)=$ $H(x, y)+I$, because the generators of $H$ and $I$ do not involve the indeterminates $x$ and $y$. It suffices then to show that for $1 \leqq i, j \leqq k$, $z\left(x_{i} y_{j}-x_{j} y_{i}\right) \in J$, where $z$ denotes $x$ or $y$. But

$$
x\left(x_{i} y_{j}-x_{j} y_{i}\right)=x_{i}\left(x y_{j}-x_{j} y\right)-x_{j}\left(x y_{i}-x_{i} y\right) \in J,
$$

and

$$
y\left(x_{i} y_{j}-x_{j} y_{i}\right)=y_{i}\left(x y_{j}-x_{j} y\right)-y_{j}\left(x y_{i}-x_{i} y\right) \in J,
$$

as required.

$I+(x, y)$ is clearly radical, since $I$ is. It suffices, then, to show that $J+H$ is radical. Thus, we have reduced to the case where $H \subset I$.

If we localize at $K[x, y]-\{0\}$, then the image of $J$ is radical. In fact, in the quotient we have that $y_{j}$ is identified with $c x_{j}$ for $1 \leqq j \leqq k$, where $c=y / x \in K(x, y)$, so that the residue class ring is isomorphic to a quotient of $K(x, y)\left[x_{1}, \cdots, x_{s}, y_{k+1}, \cdots, y_{s}\right]$ by an ideal generated by a subset of the 2 by 2 minors of the matrix

$$
\left(\begin{array}{ll}
x_{1} \cdots x_{k} & x_{k+1} \cdots x_{s} \\
c x_{1} \cdots c x_{k} & y_{k+1} \cdots y_{s}
\end{array}\right)
$$

and the claim follows from the induction hypothesis and (4.7).

It is also clear that we need only consider the case where $K$ is algebraically closed. Hence, if we let $R$ denote the reduced $K$-algebra $K\left[x_{1}, y_{1}, \cdots, x_{s}, y_{s}\right] / I$, it is evident that we need only establish the following lemma:

(4.8) Let $R$ be a reduced $K$-algebra over an algebraically closed field $K$. Suppose that the 2 by 2 minors of the matrix 


$$
\left(\begin{array}{lll}
a_{1} & \cdots & a_{k} \\
b_{1} & \cdots & b_{k}
\end{array}\right)
$$

vanish, where the $a_{i}$ and $b_{i}$ are in $R$. Let $x$ and $y$ be indeterminates over $R$, and let $J$ be the ideal generated by the 2 by 2 minors of the matrix

$$
\left(\begin{array}{llll}
x & a_{1} & \cdots & a_{k} \\
y & b_{1} & \cdots & b_{k}
\end{array}\right)
$$

in $R[x, y]$. If the image of $J$ in the localization $R[x, y]$ at $K[x, t]-\{0\}$ is radical, then $J$ is radical in $R[x, y]$.

Proof. $R[x, y]$ is a graded $R$-algebra ( $x$ and $y$ have degree 1$)$, and $J$ is generated by 1 -forms. Hence, $V \bar{J}$ is a homogeneous ideal. Suppose that $\sqrt{J} \neq J$ and let $F$ be a form of $R[x, y]$ in $\sqrt{J}-J$. Let $P=J:(F)$. Then $P$ is a homogeneous ideal, so that $P \cap K[x, y]$ is homogeneous. After localization at $K[x, y]-\{0\}, J$ expands to a radical ideal, so that we can assume that $P$ contains a nonzero form of positive degree in $K[x, y]$. Since $K$ is algebraically closed, we can write this form as a product $L_{1} \cdots L_{m}$, where the $L_{i}$ are 1-forms in $K[x, y]$. By replacing $F$ by $L_{1} \cdots L_{t} F$ for a suitable $t, 0 \leqq t \leqq m-1$, we can assume that $F \notin J$ but $L F \in J$ for a suitable 1 - form $L$. By a linear change of coordinates on $x, y$, we can suppose that $L=x$. By replacing $F$ by a suitable power, we can also assume that $F \notin J$ but $F^{2} \in J$. Let $\operatorname{deg} F=d$.

We can write $F=F_{1} x+r_{0} y^{d}$. Since $x F, F^{2} \in J$, we also have that $r_{0} y^{d} F \in J$. Since $x F \in J$, we have that

$$
x F=\sum_{i=1}^{k}\left(G_{i} x^{2}+s_{i} x y^{d-1}+t_{i} y^{d}\right)\left(b_{i} x-a_{i} y\right)
$$

where the $G_{i}$ are forms of degree $d-2$ and the $s_{i}$ and $t_{i}$ are in $R$. It follows that

$$
\sum_{i=1}^{k} t_{i} a_{i}=0
$$

and that

$$
r_{0}=\sum_{i=1}^{k} t_{i} b_{i}-\sum_{i=1}^{k} s_{i} a_{i} .
$$

Let $b=\sum_{i} t_{i} b_{i}$. Then $b \in\left(r_{0}\right)+\left(a_{1}, \cdots, a_{k}\right)$, so that

$$
b^{2} \in\left(r_{0}^{2}\right)+\left(a_{1}, \cdots, a_{s}\right) \text {. }
$$

Since $r_{0} y^{d} F \in J$, we have that

$$
r_{0} y^{d} F=\sum_{i=1}^{k}\left(H_{i} x+u_{i} y^{2 d-1}\right)\left(b_{i} x-a_{i} y\right),
$$


where the $H_{i}$ are forms of degree $2 d-2$ and the $u_{i} \in R$. If we equate coefficients of $y^{2 d}$, we find that $r_{0}^{2} \in\left(a_{1}, \cdots, a_{k}\right)$, and hence $b^{2} \in\left(a_{1}, \cdots, a_{k}\right)$. Since $R$ is reduced, if $b \neq 0$ we can find a prime $Q$ not containing $b$. Let' denote reduction modulo $Q$. Since $b^{\prime 2} \neq 0$, we have that the the row

$$
\left(a_{1}^{\prime} \cdots a_{k}^{\prime}\right)
$$

is not 0 . Since the 2 by 2 minors of

$$
\left(\begin{array}{lll}
a_{1}^{\prime} & \cdots & a_{k}^{\prime} \\
b_{1}^{\prime} & \cdots & b_{k}^{\prime}
\end{array}\right)
$$

vanish, there is a constant $c$ in the fraction field of the domain $R / Q$ such that $\left(b_{1}^{\prime} \cdots b_{k}^{\prime}\right)=c\left(a_{1}^{\prime} \cdots a_{k}^{\prime}\right)$. Then since $\sum_{i} t_{i}^{\prime} a_{i}^{\prime}=0$, we have that $b^{\prime}=\sum_{i} t_{i}^{\prime} b_{i}^{\prime}=c \sum_{i} t_{i}^{\prime} a_{i}^{\prime}=0$, a contradiction.

Hence, we must have $b=0$, i.e. $\sum_{i} t_{i} b_{i}=0$. But then $\left(^{*}\right)$ yields:

$$
F=\sum_{i}\left(G_{i} x+s_{i} y^{d-1}\right)\left(b_{i} x-a_{i} y\right) \in J,
$$

and we are done.

\section{REFERENCES}

1. J. Fogarty, Invariant Theory, W. A. Benjamin, N. Y., 1969.

2. A. Grothendieck (with J. Dieudonné), Eléments de géométrie algébrique. IV. Étude locale des schémas et des morphismes de schémas, (Seconde partie.) Publ. I. H. E. S. No. 24 (1965).

3. M. Hochster, Grassmannians and their Schubert subvarieties are arithmetically Cohen-Macaulay, to appear, J. Algebra.

4. M. Hochster and J. A. Eagon, A class of perfect determinantal ideals, Bull. Amer. Math. Soc., 76 (1970), 1026-1029.

5. - Cohen-Macaulay rings, invariant theory, and the generic perfection of determinantal loci, Amer. J. Math., 93 (1971), 1020-1059.

6. B. Kostant, Lie group representations on polynomial rings, Amer. J. Math., 85 (1963), 327-404.

7. M. Nagata, Local Rings, Interscience, N. Y.., 1962.

8. H. Weyl, The Classical Groups, Princeton University Press, Princeton, 1946.

Received October 13, 1971. Research supported in part by NSF grant GP-29224X.

UNIVERSITY OF MINNESOTA 


\section{PACIFIC JOURNAL OF MATHEMATICS}

\section{EDITORS}

\section{H. SAMELSON}

Stanford University

Stanford, California 94305

C. R. Новву

University of Washington Seattle, Washington 98105

\section{J. DuGundJI}

Department of Mathematics University of Southern California Los Angeles, California 90007

RICHARD ARENS

University of California Los Angeles, California 90024

\section{ASSOCIATE EDITORS}
E. F. BECKENBACH
B. H. NeumanN
F. WOLF
K. YoSHIDA

\section{SUPPORTING INSTITUTIONS}

\author{
UNIVERSITY OF BRITISH COLUMBIA \\ CALIFORNIA INSTITUTE OF TECHNOLOGY \\ UNIVERSITY OF CALIFORNIA \\ MONTANA STATE UNIVERSITY \\ UNIVERSITY OF NEVADA \\ NEW MEXICO STATE UNIVERSITY \\ OREGON STATE UNIVERSITY \\ UNIVERSITY OF OREGON \\ OSAKA UNIVERSITY
}

\author{
UNIVERSITY OF SOUTHERN CALIFORNIA \\ STANFORD UNIVERSITY \\ UNIVERSITY OF TOKYO \\ UNIVERSITY OF UTAH \\ WASHINGTON STATE UNIVERSITY \\ UNIVERSITY OF WASHINGTON \\ $*{ }^{*}$
AMERICAN MATHEMATICAL SOCIETY
NAVAL WEAPONS CENTER
}

The Supporting Institutions listed above contribute to the cost of publication of this Journal, but they are not owners or publishers and have no responsibility for its content or policies.

Mathematical papers intended for publication in the Pacific Journal of Mathematics should be in typed form or offset-reproduced, (not dittoed), double spaced with large margins. Underline Greek letters in red, German in green, and script in blue. The first paragraph or two must be capable of being used separately as a synopsis of the entire paper. The editorial "we" must not be used in the synopsis, and items of the bibliography should not be cited there unless absolutely necessary, in which case they must be identified by author and Journal, rather than by item number. Manuscripts, in duplicate if possible, may be sent to any one of the four editors. Please classify according to the scheme of Math. Rev. Index to Vol, 39. All other communications to the editors should be addressed to the managing editor, Richard Arens, University of California, Los Angeles, California, 90024.

50 reprints are provided free for each article; additional copies may be obtained at cost in multiples of 50 .

The Pacific Journal of Mathematics is issued monthly as of January 1966. Regular subscription rate: $\$ 48.00$ a year (6 Vols., 12 issues). Special rate: $\$ 24.00$ a year to individual members of supporting institutions.

Subscriptions, orders for back numbers, and changes of address should be sent to Pacific Journal of Mathematics, 103 Highland Boulevard, Berkeley, California, 94708.

PUBLISHED BY PACIFIC JOURNAL OF MATHEMATICS, A NON-PROFIT CORPORATION

Printed at Kokusai Bunken Insatsusha (International Academic Printing Co., Ltd.), 270, 3-chome Totsuka-cho, Shinjuku-ku, Tokyo 160, Japan. 


\section{Pacific Journal of Mathematics}

\section{Vol. 44, No. $2 \quad$ June, 1973}

Tsuyoshi Andô, Closed range theorems for convex sets and linear liftings . . . . . . 393

Richard David Bourgin, Conically bounded sets in Banach spaces . . . . . . . . . 411

Robert Jay Buck, Hausdorff dimensions for compact sets in $R^{n} \ldots \ldots \ldots \ldots \ldots \ldots . \ldots 421$

Henry Cheng, A constructive Riemann mapping theorem ................ 435

David Fleming Dawson, Summability of subsequences and stretchings of

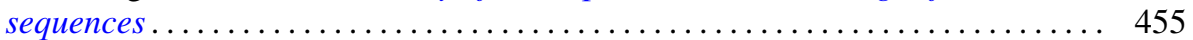

William Thomas Eaton, A two sided approximation theorem for 2-spheres ....... 461

Jay Paul Fillmore and John Herman Scheuneman, Fundamental groups of compact complete locally affine complex surfaces ....................... 487

Avner Friedman, Bounded entire solutions of elliptic equations . . . . . . . . . . . 497

Ronald Francis Gariepy, Multiplicity and the area of an $(n-1)$ continuous

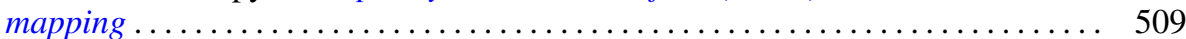

Andrew M. W. Glass, Archimedean extensions of directed interpolation groups . . . . 515

Morisuke Hasumi, Extreme points and unicity of extremum problems in $H^{1}$ on

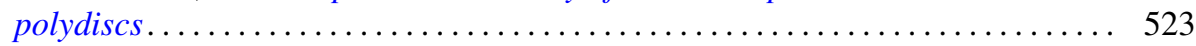

Trevor Ongley Hawkes, On the Fitting length of a soluble linear group . . . . . . 537

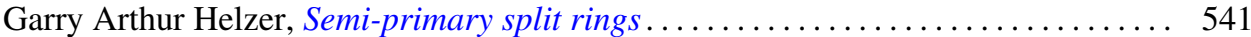

Melvin Hochster, Expanded radical ideals and semiregular ideals . . . . . . . . . 553

Keizō Kikuchi, Starlike and convex mappings in several complex variables . . . . . . 569

Charles Philip Lanski, On the relationship of a ring and the subring generated by its

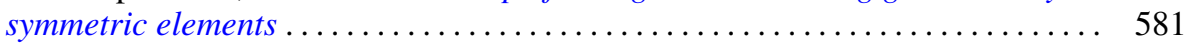

Jimmie Don Lawson, Intrinsic topologies in topological lattices and semilattices ........................................... 593

Roy Bruce Levow, Counterexamples to conjectures of Ryser and de Oliveira ...... 603

Arthur Larry Lieberman, Some representations of the automorphism group of an infinite continuous homogeneous measure algebra ..........

William George McArthur, $G_{\delta}$-diagonals and metrization theorems $\ldots .$.

James Murdoch McPherson, Wild arcs in three-space. II. An invariant of

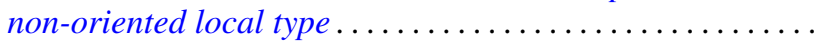

H. Millington and Maurice Sion, Inverse systems of group-valued measures ...

C. Edward Moore, Concrete semispaces and lexicographic separation of convex

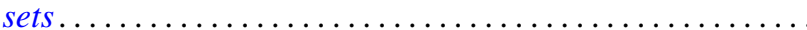

Jingyal Pak, Actions of torus $T^{n}$ on $(n+1)$-manifolds $M^{n+1}$.

Merrell Lee Patrick, Extensions of inequalities of the Laguerre and Turán type . . . . 675

Harold L. Peterson, Jr., Discontinuous characters and subgroups of finite index. . . . 683

S. P. Philipp, Abel summability of conjugate integrals . . . . . . . . . . . . . 693

R. B. Quintana and Charles R. B. Wright, On groups of exponent four satisfying an

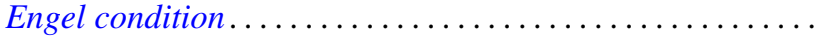

Marlon C. Rayburn, On Hausdorff compactifications. . . . . . . . . .

Martin G. Ribe, Necessary convexity conditions for the Hahn-Banach theorem in

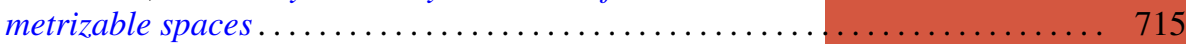

Ryōtarō Satō, On decomposition of transformations in infinite measure spaces .... 733

Peter Drummond Taylor, Subgradients of a convex function obtained from a

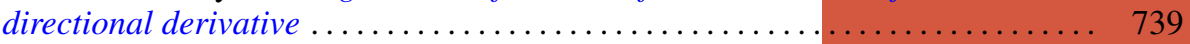

James William Thomas, A bifurcation theorem for $k$-set contractions . . . . . . . . 749 Clifford Edward Weil, A topological lemma and applications to real functions . . . . 757

Stephen Andrew Williams, A nonlinear elliptic boundary value problem . . ....... 767

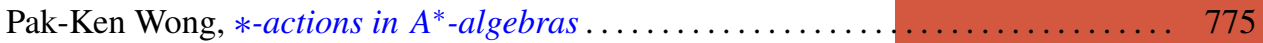

